\title{
Mortalidad infantil en la Cuenca Matanza Riachuelo. Comparación con la Ciudad de Buenos Aires, la provincia de Buenos Aires y la Argentina (de 2010 a 2017) Infant mortality in the Matanza-Riachuelo River Basin. Comparison with the Autonomous City of Buenos Aires, the province of Buenos Aires, and Argentina (2010-2017)
}

\author{
Dra. Juliana Z. Finkelstein ${ }^{a, b, c}$, Dra. María O. Codebó Ramalho Luz ${ }^{a}$, \\ Lic. Lidia E. Feiock ${ }^{a}$ Lic. Giselle Della Rosa ${ }^{a, b}$ y Dra. Susana García ${ }^{a, c}$
}

\section{RESUMEN}

Introducción. La tasa de mortalidad infantil (TMI) es un indicador de salud y de condiciones socioeconómicas, ambientales y sanitarias. Basurales a cielo abierto y desechos cloacales e industriales hacen de la Cuenca Matanza Riachuelo (CMR) la más contaminada de Argentina.

Objetivo. Analizarla mortalidad infantil(MI)en laCMRen comparación con Argentina, provincia de Buenos Aires (PBA) y Ciudad Autónoma de Buenos Aires (CABA), y la evolución entre los años 2010 y 2017.

Población y métodos. Estudio descriptivo a partir de datos del Ministerio de Salud de la Nación. Resultados. En 2017, la TMI en la CMR fue del 9,4\%o; en Argentina, del 9,3\%; en PBA, del 9,4 \%o, y, en CABA, del 6,9\%. Entre 2010 y 2017, se observó una disminución de la TMI en la CMR del 20,6\%, similar a PBA y Argentina. En CABA, no hubo descenso.

En las 4 jurisdicciones, las afecciones perinatales representaron la primera causa de muerte, seguidas por las malformaciones congénitas y las enfermedades respiratorias. Solo para enfermedad respiratoria, en la CMR, el riesgo fue 4 veces mayor que en CABA (riesgo relativo: 3,9; intervalo de confianza del $95 \%: 1,4-10,7)$.

Conclusión. La estructura de causas, la evolución y el riesgo de $\mathrm{MI}$ en la CMR, Argentina y PBA fueron similares entre 2010 y 2017. CABA presentó una TMI menor que CMR, pero no mostró descensos. El riesgo de MI en la CMR fue mayor que en CABA a expensas del mayor riesgo por enfermedad respiratoria.

Palabras clave: mortalidad infantil, contaminación ambiental, salud pública.

http:/ / dx.doi.org/10.5546/aap.2020.313

Texto completo en inglés:

http:/ / dx.doi.org/10.5546/ aap.2020.eng.313

Cómo citar: Finkelstein JZ, Codebó Ramalho Luz MO, Feiock LE, Della Rosa G, García S. Mortalidad infantil en la Cuenca Matanza Riachuelo. Comparación con la Ciudad de Buenos Aires, la provincia de Buenos Aires y la Argentina (de 2010 a 2017). Arch Argent Pediatr 2020;118(5):313-319.

\section{INTRODUCCIÓN}

La tasa de mortalidad infantil (TMI) es un indicador del estado de salud de la población en general y de los niños menores de 1 año en particular. Además, es un indicador de las condiciones socioeconómicas, ambientales y de accesibilidad y calidad de los sistemas de servicios de salud. ${ }^{1}$

En septiembre de 2015, los Estados miembros de la Organización de Naciones Unidas aprobaron la Agenda 2030 para el Desarrollo Sostenible para poner fin a la pobreza, luchar contra la desigualdad y la injusticia, y hacer frente al cambio climático. ${ }^{2}$ Entre sus objetivos, plantea la necesidad de reducir la mortalidad de los niños menores de 5 años. Aproximadamente, el $26 \%$ de la mortalidad y más del $40 \%$ del total de las enfermedades de los menores de 5 años en el mundo son atribuidas a factores ambientales. ${ }^{3,4}$

A pesar de los avances en el cuidado de la salud de las últimas décadas en el mundo, millones de niños continúan muriendo por causas reducibles. Esto evidencia dificultades en la calidad y la accesibilidad a servicios básicos de salud, la situación nutricional, al acceso al agua segura y al saneamiento ambiental..$^{5-7}$

El río Matanza-Riachuelo tiene 64 kilómetros de longitud; fluye desde el oeste de la provincia de Buenos Aires (PBA) hacia el estuario del Río de la Plata, y su cuenca 
abarca $2200 \mathrm{~km}^{2}$ (Figura 1). La presencia de basurales a cielo abierto, el vertido de desechos cloacales e industriales de más de 25000 establecimientos industriales y actividades de servicios son fuente de contaminación del agua (superficial y subterránea), el suelo y el aire de la Cuenca Matanza Riachuelo (CMR). ${ }^{8-10}$

La CMR es la más contaminada de la Argentina, una de las 5 más contaminadas de América Latina y una de las 30 más contaminadas del mundo. ${ }^{11}$ Con más de 4 millones de habitantes, es una zona densamente poblada donde coexiste la exposición a la contaminación con deficientes condiciones habitacionales e importantes vulnerabilidades socioeconómicas. ${ }^{12}$

En septiembre de 2017, la Dirección de Salud y Educación Ambiental (DSyEA) de la Autoridad de la Cuenca Matanza Riachuelo (ACUMAR), junto con la Asociación Toxicológica Argentina, la Sociedad Argentina de Pediatría y la Sociedad Argentina de Medicina, establecieron el listado de enfermedades relacionadas con la exposición ambiental, con la finalidad de pesquisar y vigilar su presencia en los barrios de la CMR. Entre estos eventos, se encuentran la prematurez y el bajo peso al nacer, así como las malformaciones congénitas y las enfermedades respiratorias. Estas causas explican más del $85 \%$ de la mortalidad infantil en la Argentina. ${ }^{13}$

El objetivo de este trabajo fue analizar la estructura de causas de la mortalidad infantil en la CMR en comparación con la Argentina, la PBA y la Ciudad Autónoma de Buenos Aires (CABA), y la evolución entre los años 2010 y 2017.

\section{POBLACIÓN Y MÉTODOS}

Se realizó un estudio descriptivo de la mortalidad infantil (defunciones de menores de un año) en la CMR en comparación a la Argentina, la PBA y la CABA a partir de la base de Estadísticas Vitales de la Dirección de Estadísticas e Información de Salud (DEIS) del Ministerio de Salud de la Nación del año 2010 al 2017.

Cada nacimiento y cada defunción ocurridos en la Argentina son registrados en un certificado legal y un informe estadístico. Los informes estadísticos de nacidos vivos (IENV) y de defunciones (IED) son consolidados a nivel jurisdiccional y nacional. Cada año, la DEIS del Ministerio de Salud de la Nación consolida, analiza y publica los principales resultados sobre las Estadísticas Vitales a partir de los informes estadísticos correspondientes. ${ }^{14}$

En este análisis, se incluyeron los datos de

Figura 1. Ubicación de la Cuenca Matanza Riachuelo en la región noroeste de la provincia de Buenos Aires y sur de la Ciudad Autónoma de Buenos Aires

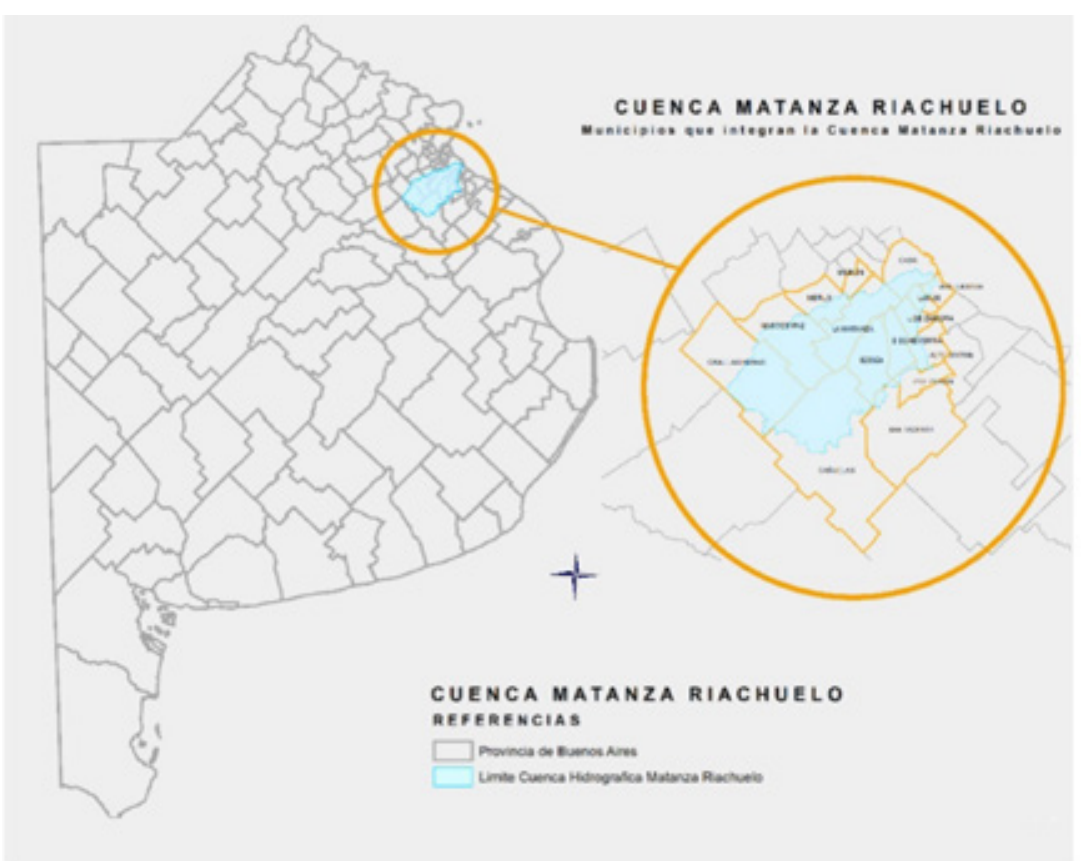

Fuente: Autoridad de la Cuenca Matanza Riachuelo (ACUMAR). 
todos los nacidos vivos y todas las defunciones de niños menores de un año ocurridas en la CMR, la Argentina, la PBA y la CABA registradas según la jurisdicción de residencia de la madre. Para analizar la mortalidad infantil y la estructura de causas, se utilizaron tasas, y no los números absolutos, para poder realizar comparaciones con otras poblaciones y a lo largo del tiempo. Se calcularon la TMI, la TMI específica según causas y la mortalidad proporcional según causas, todas variables cuantitativas continuas. ${ }^{15}$

La TMI relacionó las defunciones infantiles ocurridas durante un año con el número de nacidos vivos registrados en el transcurso del mismo año en la jurisdicción correspondiente y se expresó cada 1000 nacidos vivos. ${ }^{16}$ La TMI según causas comprendió las defunciones de los menores de un año por una causa determinada en relación con el número de nacidos vivos registrados en el transcurso del mismo año en dicha jurisdicción y se expresó cada 1000 nacidos vivos. ${ }^{17}$ La mortalidad proporcional según causas expresa la relación entre las defunciones infantiles por una causa determinada y la totalidad de las muertes infantiles, expresada como porcentaje.

La información correspondiente a la CMR se calculó integrando los datos de 14 municipios de PBA y las cuatro comunas de CABA que la componen (grado máximo de desagregación de la información de la DEIS). Los 14 municipios de PBA que integran la CMR son Almirante Brown, Avellaneda, Cañuelas, Esteban Echeverría, General Las Heras, La Matanza, Lanús, Lomas de Zamora, Marcos Paz, Merlo, Morón, Presidente Perón y San Vicente. Las comunas de CABA que componen la CMR son la 4, la 7, la 8 y la 9.

Se calculó el riesgo relativo (RR) (intervalo de confianza-IC- del $95 \%$ ) de morir en el primer año de vida tomando como evento la defunción infantil y como exposición la residencia de la madre en la CMR, comparado con la CABA, la PBA y la Argentina, y se analizó el riesgo de muerte según las principales causas de defunción infantil en la CMR con respecto a las mismas jurisdicciones. Dado que el RR es una medida del riesgo de que un evento (defunción infantil) suceda en un grupo expuesto (residencia materna en la CMR) en comparación con el riesgo de que el mismo evento suceda en otro grupo de no expuestos (hijos de madres no residentes en la CMR), para el cálculo de este indicador en las jurisdicciones de comparación, se excluyeron las defunciones y los nacidos vivos con residencia materna en las comunas o municipios que conforman la CMR. ${ }^{18}$

Se utilizaron los softwares de procesamiento de datos Microsoft Excel y Epidat 4.2. El estudio se realizó sobre la base de fuentes secundarias, sin utilizar datos personales, por lo cual no requirió consentimiento informado ni aprobación del protocolo por el Comité de Ética.

\section{RESULTADOS}

En el año 2017, se registró, en la CMR, una TMI del 9,45\%; en la Argentina, del 9,33\%o, $\mathrm{y}$, en la PBA, del 9,4\%o. No hubo diferencias estadísticamente significativas entre la PBA y la Argentina con la CMR (Tabla 1). En la CABA, la TMI fue del 6,92\% y el riesgo de muerte infantil fue el $40 \%$ menor que en la CMR (RR: 1,4; IC $95 \%$ : 1,2-1,6) (Tabla 1 y 3).

En el análisis de la evolución de la TMI a lo largo del período de 2010 a 2017, se observó una evolución descendente en la CMR (disminución del 20,6\%), en la Argentina (disminución del $21,5 \%$ ) y en la PBA (disminución del $21,6 \%$ ).

TABla 1. Nacidos vivos, defunciones de menores de un año y tasa de mortalidad infantil, según jurisdicción de residencia materna. Año 2017. Cuenca Matanza Riachuelo, Ciudad Autónoma de Buenos Aires, provincia de Buenos Aires y la Argentina

\begin{tabular}{lcccc}
\hline $\begin{array}{l}\text { Jurisdicción de } \\
\text { residencia materna }\end{array}$ & Nacidos vivos & $\begin{array}{c}\text { Defunciones de menores } \\
\text { de un año }\end{array}$ & TMI (por 1000 NV) & $\begin{array}{c}\text { IC 95 \%, } \\
\text { TMI }\end{array}$ \\
\hline CMR & 99715 & 942 & 9,45 & $8,85-10,07$ \\
Argentina & 704609 & 6579 & 9,33 & $9,11-9,34$ \\
PBA & 262721 & 2471 & 9,40 & $9,04-9,78$ \\
CABA & 35840 & 248 & 6,92 & $6,08-7,84$ \\
\hline
\end{tabular}

CMR: Cuenca Matanza Riachuelo; CABA: Ciudad Autónoma de Buenos Aires; PBA: provincia de Buenos Aires; TMI: tasa de mortalidad infantil; NV: nacidos vivos; IC: intervalo de confianza.

Fuente: Elaboración propia sobre la base de datos de la Dirección de Estadísticas e Información de Salud del Ministerio de Salud de la Nación. Año 2017. CMR, CABA, PBA y la Argentina. 
En la CABA, el distrito con menor TMI de los analizados, se observaron oscilaciones a lo largo del período: 2010 comenzó y 2017 terminó con valores similares (el $7 \%$ y el 6,9\%o, respectivamente) (Figura 2).

$\mathrm{Al}$ analizar el peso relativo de cada grupo de causas de la mortalidad infantil en la CMR en el año 2017, la mayor parte de las defunciones registradas (el $49 \%$ ) correspondieron a las afecciones originadas en el período perinatal (principalmente, bajo peso al nacer y prematurez). En segundo lugar, estuvieron las malformaciones congénitas (el $28 \%$ ). La tercera causa fueron las enfermedades respiratorias (el 6,8\%); luego las

FIgura 2. Evolución de la tasa de mortalidad infantil según jurisdicción de residencia de la madre. Años 2010-2017. Cuenca Matanza Riachuelo, Ciudad Autónoma de Buenos Aires, provincia de Buenos Aires y la Argentina

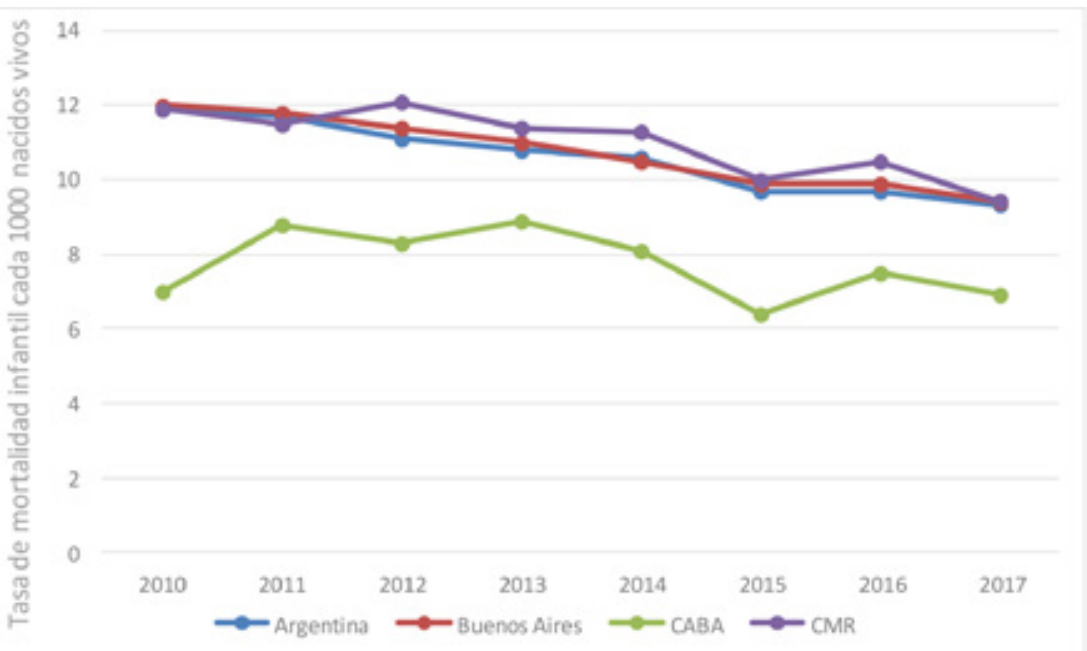

CABA: Ciudad Autónoma de Buenos Aires; CMR: Cuenca Matanza Riachuelo.

Fuente: Elaboración propia sobre la base de datos de la Dirección de Estadísticas e Información de Salud del Ministerio de Salud de la Nación. Años 2010-2017. CMR, CABA, PBA y la Argentina.

TABLA 2. Mortalidad Infantil según causa y según jurisdicción de residencia de la madre. Año 2017. Cuenca Matanza Riachuelo, Ciudad Autónoma de Buenos Aires, provincia de Buenos Aires y la Argentina

\begin{tabular}{lccccccc}
\hline & Total & $\begin{array}{c}\text { Afecciones } \\
\text { perinatales }\end{array}$ & $\begin{array}{c}\text { Malformaciones } \\
\text { congénitas }\end{array}$ & $\begin{array}{c}\text { Enfermedades } \\
\text { respiratorias }\end{array}$ & $\begin{array}{c}\text { Mal } \\
\text { definidas }\end{array}$ & $\begin{array}{c}\text { Otras } \\
\text { causas }\end{array}$ \\
\hline CMR & Defunciones infantiles & 942 & 462 & 264 & 64 & 56 & 96 \\
& TMI (por 1000 NV) & 9,45 & 4,63 & 2,64 & 0,64 & 0,56 & 0,96 \\
& IC 95 \%, TMI & $8,85-10,07$ & $4,22-5,07$ & $2,34-2,98$ & $0,49-0,82$ & $0,42-0,73$ & $0,78-1,18$ \\
& Mortalidad proporcional & $100 \%$ & $49 \%$ & $28 \%$ & $6,80 \%$ & $6,00 \%$ & $10,20 \%$ \\
Argentina & Defunciones infantiles & 6579 & 3383 & 1864 & 402 & 294 & 636 \\
& TMI (por 1000 NV) & 9,33 & 4,8 & 2,64 & 0,57 & 0,42 & 0,9 \\
& IC 95 \%, TMI & $9,11-9,34$ & $4,64-4,81$ & $2,58-2,77$ & $0,52-0,63$ & $0,37-0,47$ & $0,83-0,98$ \\
& Mortalidad proporcional & $100 \%$ & $51,40 \%$ & $28,30 \%$ & $6,10 \%$ & $4,50 \%$ & $9,70 \%$ \\
\multirow{2}{*}{ PBA } & Defunciones infantiles & 2471 & 1176 & 730 & 175 & 146 & 244 \\
& TMI (por 1000 NV) & 9,4 & 4,47 & 2,78 & 0,67 & 0,55 & 0,93 \\
& IC 95 \%, TMI & $9,04-9,78$ & $4,22-4,74$ & $2,58-2,99$ & $0,57-0,77$ & $0,47-0,65$ & $0,82-1,05$ \\
& Mortalidad proporcional & $100 \%$ & $47,60 \%$ & $29,50 \%$ & $7,10 \%$ & $5,90 \%$ & $9,90 \%$ \\
CABA & Defunciones infantiles & 248 & 133 & 77 & 15 & 3 & 20 \\
& TMI (por 1000 NV) & 6,92 & 3,71 & 2,14 & 0,42 & 0,08 & 0,55 \\
& IC 95 \%, TMI & $6,08-7,84$ & $3,11-4,39$ & $1,69-2,68$ & $0,23-0,69$ & $0,02-0,25$ & $0,34-0,86$ \\
& Mortalidad proporcional & $100 \%$ & $53,60 \%$ & $31,10 \%$ & $6 \%$ & $1,20 \%$ & $8,10 \%$ \\
\hline
\end{tabular}

CMR: Cuenca Matanza Riachuelo; CABA: Ciudad Autónoma de Buenos Aires; PBA: provincia de Buenos Aires;

TMI: tasa de mortalidad infantil; NV: nacidos vivos; IC: intervalo de confianza.

Fuente: Elaboración propia sobre la base de datos de la Dirección de Estadísticas e Información de Salud del Ministerio de Salud de la Nación. Año 2017. CMR, CABA, PBA y la Argentina. 
defunciones con causa básica mal definida (el $6 \%$ ) y el resto de las causas (el 10,2 \%) (Tabla 2).

La estructura de causas de mortalidad infantil fue similar en la CMR, la Argentina, la PBA y la CABA. En las 4 jurisdicciones, las afecciones originadas en el período perinatal representaron la primera causa de muerte, seguidas por las defunciones por malformaciones congénitas y las enfermedades respiratorias. Estas 3 causas, relacionadas con la exposición ambiental, representaron, en 2017, el 83,8 \% de las defunciones infantiles de la CMR, el 85,9\% en la Argentina, el $84,2 \%$ en la PBA y el $90,7 \%$ en la CABA (Tabla 2).

La TMI por afecciones perinatales en la CMR (el 4,6\%o) fue más alta que la de la CABA (el $3,7 \%$ ) y la PBA (el $4,5 \%$ ), aunque inferior a la del total del país (el 4,8\%o). Estas diferencias no tuvieron significación estadística (Tablas 2 y 3).

La segunda causa fueron las malformaciones congénitas, con una tasa del 2,6\% para CMR, igual que en la Argentina, más alta que en la CABA $($ el $2,1 \%$ ) y menor que en la PBA (el 2,8\%o). Tampoco estas diferencias tuvieron significación estadística (Tablas 2 y 3).

Las defunciones por enfermedades respiratorias en la CMR presentaron una TMI específica del 0,6\%o, igual que en la Argentina, más alta que en la CABA (el $0,4 \%$ ) y menor que en la PBA (el 0,7 \%o). Al comparar el riesgo de muerte infantil por enfermedades respiratorias, en la CMR, fue similar al de la Argentina y la PBA, pero 4 veces mayor que en la CABA (RR 3,9; IC $95 \%$ : 1,4-10,7) (Tablas 2 y 3).

\section{DISCUSIÓN}

La disminución de la TMI en la CMR en el período analizado (años 2010-2017) fue del 20,6\%, similar a la PBA (el 21,6\%) y la Argentina (el 21,5\%), y también al descenso observado en América Latina y el Caribe (el 19,9\%) y en el mundo (el 20,8\%). ${ }^{19}$ Cabe mencionar que, en el período analizado, en la CABA, no se pudo observar este descenso.

En el año 2017, fallecieron, en el mundo, 5,4 millones de niños menores de 5 años, y 4,1 millones ocurrieron durante el primer año de vida (el $76 \%$ de las defunciones de menores de 5 años). Las principales causas fueron la prematurez, la neumonía, las complicaciones durante el parto, las anomalías congénitas, las enfermedades diarreicas, sepsis neonatal y malaria estrechamente relacionadas con el medioambiente..$^{20-22}$

El parto prematuro se asocia con enfermedades y discapacidad durante toda la vida. La prematurez, que representó el $18 \%$ de las muertes infantiles, se ha asociado a la exposición a la contaminación atmosférica, la contaminación del aire en interiores, el humo de tabaco, las sustancias químicas y la falta de acceso adecuado al agua segura, la falta de saneamiento y de higiene. ${ }^{7,22}$

Las infecciones de las vías respiratorias representaron el $16 \%$ de las muertes. ${ }^{22} \mathrm{La}$ Organización Mundial de la Salud (OMS) atribuye a la contaminación del aire en interiores por el uso de combustibles sólidos para cocinar y la contaminación atmosférica, más del $50 \%$ de las infecciones de las vías respiratorias inferiores en los niños menores de cinco años en los países con ingresos medios y bajos. ${ }^{7}$

E1 $9 \%$ de las muertes de lactantes en el mundo se deben a anomalías congénitas, relacionadas con la exposición al humo de tabaco, la exposición a los plaguicidas, los disolventes orgánicos, la contaminación del aire, etc. ${ }^{7}$ Las enfermedades diarreicas provocan cerca del $8 \%$

Tabla 3. Comparación del riesgo de muerte infantil entre la Cuenca Matanza Riachuelo y la Argentina, la provincia de Buenos Aires y la Ciudad Autónoma de Buenos Aires, expresada como riesgo relativo e intervalo de confianza del $95 \%$

\begin{tabular}{lccccc}
\hline & & Total & Afecciones perinatales & Malformaciones congénitas & Enfermedades respiratorias \\
\hline \multirow{2}{*}{ Argentina } & RR & 1,01 & 0,95 & 1,14 & 1,14 \\
& IC $95 \%$ & $0,95-1,08$ & $0,86-1,05$ & $1,00-1,30$ & $0,87-1,50$ \\
PBA & RR & 1,02 & 1,07 & 0,94 & 0,91 \\
& IC $95 \%$ & $0,94-1,10$ & $0,96-1,20$ & $0,81-1,09$ & $0,67-1,24$ \\
CABA & RR & 1,4 & 1,22 & 1,21 & 3,9 \\
& IC $95 \%$ & $1,19-1,65$ & $0,98-1,53$ & $0,90-1,63$ & $1,4-10,7$ \\
\hline
\end{tabular}

CABA: Ciudad Autónoma de Buenos Aires; PBA: provincia de Buenos Aires; RR: riesgo relativo; IC: intervalo de confianza. Fuente: Elaboración propia sobre la base de datos de la Dirección de Estadísticas e Información de Salud del Ministerio de Salud de la Nación. Año 2017. CMR, CABA, PBA y la Argentina. 
del total de muertes de niños menores de cinco años, relacionadas con el acceso al agua segura, saneamiento e higiene. ${ }^{22} \mathrm{~A}$ la malaria se atribuyen más de 300000 muertes de niños menores de cinco años y se señala la preocupación por el dengue, que es la enfermedad vírica transmitida por mosquitos que se propaga más rápido en el mundo y tiene a la mitad de la población mundial en riesgo. ${ }^{7}$

Al comparar los resultados de este estudio con la situación a nivel mundial, las enfermedades respiratorias representaron, en las jurisdicciones incluidas en la CMR, menos de la mitad del peso relativo del hallado a nivel mundial; las diarreas tuvieron un peso poco relevante, y el dengue, junto con la malaria, no aparecieron como causas de muerte infantil en la Argentina, mientras que las afecciones perinatales tuvieron un peso relativo mayor en las jurisdicciones analizadas de la CMR que a nivel mundial. ${ }^{7,22}$ Independientemente del peso relativo de cada una de estas causas, las afecciones perinatales, las malformaciones congénitas, las enfermedades respiratorias e infecciosas y su estrecha relación con la exposición ambiental representaron las primeras causas de muerte infantil en la CMR, la PBA, la CABA, la Argentina y el resto del mundo. ${ }^{7,22}$

La estructura de causas de muerte en los primeros años de vida en el mundo está fuertemente determinada por la estructura de causas de las defunciones infantiles en Asia y África, que representan más del $80 \%$ de las muertes en los primeros años de vida en el mundo. ${ }^{5}$ En estos continentes, las condiciones de vida representan una mayor vulnerabilidad ante las amenazas ambientales y esto explicaría las diferencias en la estructura de las causas de muerte con respecto a lo que ocurre en la CMR en particular y en la Argentina en general.

El 24,62 \% de la población de la CMR no tiene acceso a la red de agua potable (el 16,1\% en la Argentina, el $24,9 \%$ en la PBA y el 0,4 \% en la CABA); el 52,4\% no tiene acceso a una red cloacal (el 46,9 \% en la Argentina, el 52,4 \% en la PBA y el 1,8\% en la CABA), y el 11,2\% tiene las necesidades básicas insatisfechas (el 12,5\% en la Argentina, el 11,2\% en la PBA y el $7 \%$ en la CABA). ${ }^{23,24}$ En este sentido, resulta interesante analizar el impacto de los determinantes sociales sobre los resultados de la exposición ambiental; sin embargo, en este estudio, no se pudo realizar debido a la ausencia de estos datos en los informes estadísticos correspondientes.
Es por todo esto por lo que resulta imprescindible, no solo en la CMR, sino en toda la Argentina, disminuir el riesgo de exposición ambiental y mejorar las condiciones preconcepcionales de las mujeres en edad fértil, el control precoz y adecuado del embarazo, la atención del parto en maternidades seguras y en el nivel de complejidad que corresponda según el riesgo. Además, es necesario mejorar las condiciones de vida y los controles de salud de los niños pequeños.

Entre las debilidades que presenta este trabajo, debe señalarse que se analizaron datos publicados por la DEIS, provenientes del Sistema de Estadísticas Vitales, basados en los IENV e IED, y que el subregistro del evento (nacimiento o defunción) o la incorrecta identificación de la causa de muerte en los IED podrían afectar los resultados del análisis. Sin embargo, entre las fortalezas, cabe señalar que más del 99,5\% de los nacimientos en nuestro país son institucionales y que la confección simultánea del IED y el certificado de defunción es obligatoria para la inhumación de los cuerpos, lo cual disminuye la posibilidad de subregistro de estos eventos.

\section{CONCLUSIÓN}

El riesgo de MI y su estructura de causas fue similar en la CMR, la Argentina y la PBA. El riesgo de MI en la CMR fue mayor que en la CABA. El riesgo de MI según causas solo evidenció mayor riesgo de MI por enfermedades respiratorias en la CMR que en la CABA. La disminución de la TMI fue similar entre 2010 y 2017 en la CMR, la PBA y la Argentina. La CABA no mostró descensos en su TMI en el período analizado.

\section{REFERENCIAS}

1. Finkelstein J, Duhau M, Speranza A, Marconi E, et al. Evolución de la mortalidad infantil en Argentina en el marco de los Objetivos del Desarrollo del Milenio. Arch Argent Pediatr. 2016; 114(3):216-22.

2. Programa de las Naciones Unidas para el desarrollo. Objetivos de Desarrollo sostenible. 2015. [Acceso: 1 de noviembre de 2019]. Disponible en: http://www.undp. org/content/ undp/es/home/ sustainable-developmentgoals.html.

3. Organización Mundial de la Salud. ¿La Herencia de Un Mundo Sostenible? Atlas Sobre Salud Infantil y Medio Ambiente. Ginebra: OMS; 2018.

4. Quiroga D, Fernández R, Paris E (comp.). Salud Ambiental Infantil: Manual para Enseñanza de Grado en Escuelas de Medicina. Buenos Aires: Ministerio de Salud de la Nación, Organización Panamericana de la Salud; 2010.

5. UNICEF, WHO, World Bank Group and United Nations. Levels and Trends in Child Mortality: Report 2019, Estimates developed by the United Nations Inter-agency 
Group for Child Mortality Estimation. New York, 2019. [Acceso: 1 de diciembre de 2019]. Disponible en: https:/ / www.unicef.org/reports / levels-and-trends-childmortality-report-2019.

6. Pronczuk-Garbino J (ed.). Children's Health and the Environment: A Global Perspective. Ginebra: OMS; 2005.

7. Organización Mundial de la Salud. ¡No contamines mi futuro! El Impacto de los factores medioambientales en la salud infantil. Ginebra: OMS; 2017.

8. Cuerpo Colegiado de ACUMAR. Informe especial de seguimiento Cuenca Matanza Riachuelo. 2003-2005. [Acceso: 1 de noviembre de 2019]. Disponible en: http:/ / cdi.mecon.gov.ar/bases/docelec/az1260.pdf.

9. Nápoli A, García Espil J. Recomposición ambiental de la Cuenca Matanza-Riachuelo. Una oportunidad Histórica que aún reclama un fuerte compromiso político y más eficiencia en la gestión. Informe Ambiental FARN. Buenos Aires: FARN; 2011. [Acceso: 6 de abril de 2020]. Disponible en: http:/ / farn.org.ar/wp-content/uploads / 2013/02 / IAF2010napoli.pdf.

10. Nápoli A. Una política de Estado para el Riachuelo. Informe Ambiental FARN. Buenos Aires: FARN; 2009. [Acceso: 6 de abril de 2020]. Disponible en: https: / / farn.org.ar/wpcontent/uploads / 2013/02/IAF2009napoli.pdf.

11. Blacksmith Institute. The world's worst polluted places. The top ten of the dirty thirty. Nueva York: Blacksmith Institute; 2007.

12. Marconi A. Mortalidad en la región de la Cuenca MatanzaRiachuelo Análisis período 2001-2009. Rev I-Salud. 2015; 10(48):49-60.

13. Sociedad Argentina de Pediatría, Sociedad Argentina de Medicina, Asociación Toxicológica Argentina D de S y EA (ACUMAR). Enfermedades relacionadas al medio ambiente en el ámbito de la Cuenca Matanza Riachuelo: Acta acuerdo de sociedades científicas. En: Estrategias de Salud Ambiental en la Cuenca Matanza Riachuelo. Buenos Aires: ACUMAR; 2018:110-5. [Acceso: 1 de noviembre de 2019]. Disponible en: http://www.acumar.gob.ar/ wp-content/uploads / 2016/12/IF-2018-53235166-APNDSYEAACUMAR-Documento-de-Salud-AP.pdf.

14. Dirección de Estadísticas e Información de Salud. Estadísticas Vitales Información Básica. 2017; Serie 5(61). Buenos Aires: DEIS; 2018. [Acceso: 6 de abril de 2020].
Disponibleen:http:/ / www.deis.msal.gov.ar/wp-content/ uploads/2019/01/Serie5Nro61.pdf.

15. Organización Panamericana de la Salud. Lineamientos básicos para el análisis de la mortalidad. Washington: OPS; 2012.

16. Dirección de Estadísticas e Información de Salud (DEIS). Definiciones y conceptos. [Acceso: 6 de abril de 2020]. Disponible en:http: / /www.deis.msal.gov.ar/index.php/ definiciones-y-conceptos/.

17. Dirección de Estadísticas e Información de Salud (DEIS). Presentación Anuario 2017. [Acceso: 6 de abril de 2020]. Disponibleen:http:/ / www.deis.msal.gov.ar/wp-content/ uploads / 2019/01/Presentacion-anuario-2017-DEIS.pdf.

18. Ortíz Z, Esandi ME, Bartman M. Epidemiología Básica y Vigilancia de la Salud. $2^{\text {da }}$ ed. Buenos Aires: MINSAL, VIGI+A, World Bank; 2004.

19. The World Bank. Data. Mortality rate, infant (per 1,000 live births). [Acceso: 6 de abril de 2020]. Disponible en: https: / / data.worldbank.org/indicator/SP.DYN.IMRT.IN.

20. Organización Mundial de la Salud. Reducción de la mortalidad en la niñez. Septiembre de 2019. [Acceso: 6 de abril de 2020]. Disponible en: https:/ / www.who.int/ es / news-room / fact-sheets / detail / children-reducingmortality.

21. UNICEF. Mortalidad infantil: ¿quéhay detrás de los datos? [Acceso: 6 de abril de 2020]. Disponible en: https: / / www. unicef.es/blog/mortalidad-infantil-que-hay-detras-de-losdatos.

22. United Nations Inter-agency Group for Child Mortality Estimation. Level \& Trends in Child Mortality. 2018. [Acceso: 6 de abril de 2020]. Disponible en: https: / / data. unicef.org/wp-content/ uploads / 2018/09/UN-IGMEChild-Mortality-Report-2018.pdf.

23. Instituto Nacional de Estadísticas y Censos. Necesidades básicas insatisfechas. Censo Nacional de Población, Hogares y Viviendas 2010. Argentina, 2010. [Acceso: 1 denoviembre de2019]. Disponibleen:https: / / www.indec.gob.ar/indec/ web / Nivel4-Tema-4-47-156.

24. Dirección de Salud y Educación Ambiental, ACUMAR. Dosier Don Juan, La Matanza. CABA. 2019. [Acceso: 6 de abril de 2020]. Disponible en http://www.acumar.gob. ar/wp-content / uploads / 2016/12/ Dosier-EISAAR-DonJuan-La-Matanza.pdf. 